\title{
Closed-form Theory of Nuclei Separation on Highly Anisotropic Surfaces
}

\author{
Feng Du and Hanchen Huang * \\ Department of Mechanical and Industrial Engineering, Northeastern University, \\ Boston, MA 02115, USA
}

\begin{abstract}
During deposition on surfaces of highly anisotropic diffusion, nucleation behaves as it would on a one-dimensional substrate, or effectively deposition in a two-dimensions (2D). This Article reports a close-form theory for nuclei separation during physical vapor deposition in 2D. In comparison and contrast, this closed-form theory agrees with existing theories in scaling, but gives the coefficient that was previously unknown. Further, this theory shows that the nuclei separation in $2 \mathrm{D}$ is an order-of-magnitude larger than in three dimensions. The insights from such comparison and contrast are critical in analyzing nucleation on surfaces of various anisotropy.
\end{abstract}

\section{Keywords:}

Nuclei separation, closed-form theory, physical vapor deposition, diffusion anisotropy

\footnotetext{
* Author to who correspondence should be made; electronic mail:

h.huang@northeastern.edu
} 


\section{Introduction}

Nucleation of atomic clusters on a solid substrate is generic in many surface processes, such as physical vapor deposition (PVD). As shown in Fig. 1, the area density of nuclei first increases with deposition time or coverage, reaches a maximum, and then decreases as some nuclei start to merge. Under glancing angle PVD on a non-wetting substrate, the merge of nuclei can be avoided and nanorods develop. As a result, the nuclei separation at the maximum area density also defines the separation of nanorods, and is called the critical separation. This quantity of critical separation has been exhaustively investigated, for example references [1, 2]. However, this line of thinking has not led to an analytical or closed-form theory of the critical separation.

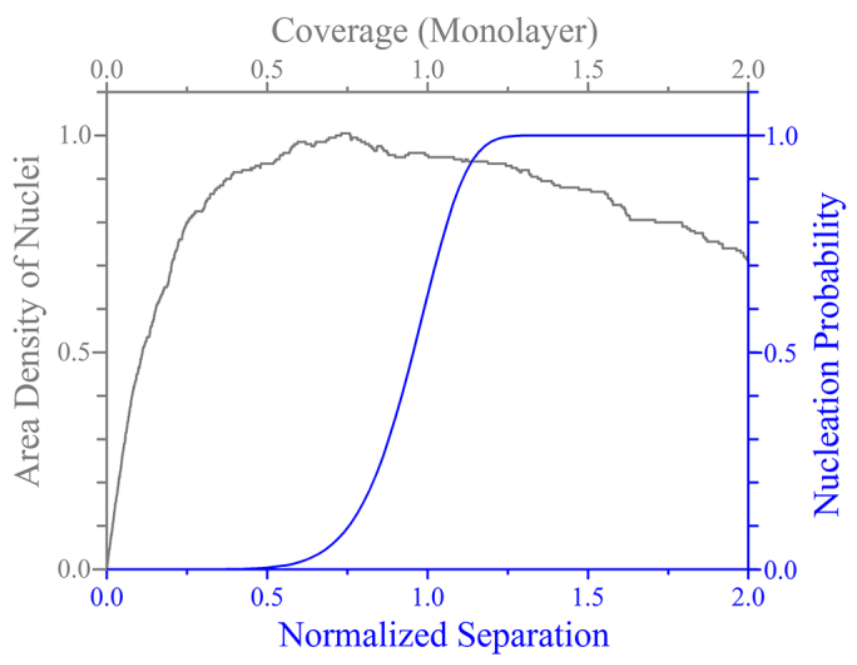

Fig. 1. Schematic of area density of nuclei, which is normalized so the maximum density is 1 , as a function of coverage; and nucleation probability as a function of separation of nuclei, which is normalized so the critical separation is 1 .

An analytical or closed-form theory of the critical nuclei separation becomes possible, as we think and formulate in a different phase space; shown in Fig. 1. In the new phase space, one variable is the separation of existing nuclei on a substrate. As the separation increases, the other variable - the nucleation probability of forming additional clusters on the substrate - goes from 0 to 1 . The critical separation is the 
separation at which the nucleation probability sharply transitions from 0 to 1 . Following this logic, we have obtained a closed-form theory of the critical separation in 3D [3], and further used it to guide the experimental realization of the smallest and well-separated nanorods from PVD [4].

It might appear illogical to develop a closed-form theory in 2D, when it is already available in 3D. However, that appearance is incorrect here. There are two good reasons for this investigation in 2D. First, surface diffusion can be highly anisotropic on some crystalline surfaces such as on the 2 x1-dimer-reconstructed $\mathrm{Si}$ $\{001\}$ surfaces [5]. In this case, nucleation is dominated by atomic diffusion along one dimension of the surface and atomic deposition along the other dimension - that is, the theory in $2 \mathrm{D}$ applies. Second, 2D atomistic simulations are still useful because of their reach to larger length and longer time scales than 3D atomistic simulations. To properly appreciate the simulation results, it is important to be able to analytically comprehend the order-of-magnitude differences between physical quantities in 2D and those in 3D.

In this Article, we first derive the closed-form theory of the critical separation of nuclei in 2D. Then we use lattice Kinetic Monte Carlo (KMC) simulations to verify the theory. By comparison and contrast of the two analytical theories in $2 \mathrm{D}$ and $3 \mathrm{D}$, we show that under typical PVD conditions the average separation of nuclei in 2D is an order of magnitude larger than in 3D.

\section{Closed-form Theory}

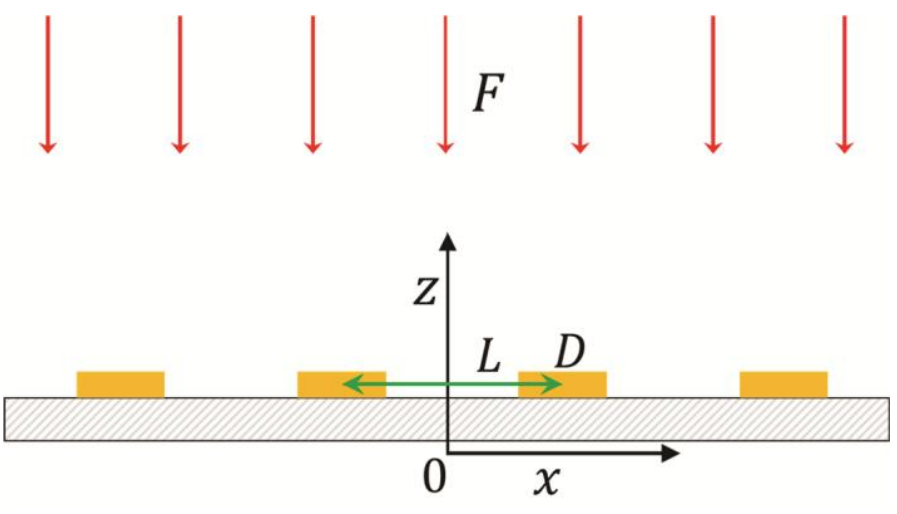


Fig. 2. Schematic of deposition in $2 \mathrm{D}$, with existing nuclei of diameter $D$ and separation $L$, and deposition rate $F$.

In deriving the theory, we consider a representative nucleation space $x \in\left[-\frac{L-D}{2}, \frac{L-D}{2}\right]$ as shown in Fig. 2. Following the same approach as in our previous report [3], we first derive the analytical expression of adatom concentration on a flat substrate. Based on the concentration, we then determine the nucleation probability, and therefore the critical separation. Due to the different time scales of deposition and diffusion, the adatom concentration $n(x)$ reaches a quasi-steady state between two deposition events $[6,7]$. Therefore, the governing diffusion equation is:

$\frac{v}{2} \nabla^{2} n(x)+F=0$

where $v$ is the adatom diffusion jump rate. The adatom concentration $n(x)$ is fractional and the length $x$ is in unit of the nearest neighbor distance (lattice unit). The deposition rate $F$ is in unit of monolayer per second $(M L / S)$. The first boundary condition is that the adatom concentration is zero near a nucleus, and the second boundary condition is that there is no net flux at $x=0$ due to symmetry. The adatom concentration under these boundary conditions is:

$n(x)=\frac{F}{v}\left[\left(\frac{L-D}{2}\right)^{2}-x^{2}\right]$

As previously shown [8], the conventional nucleation theory does not apply here because only one adatom exists on the surface most of the time. Instead, the nucleation probability per unit length follows the lone-adatom-model (LAM) and is [9]:

$p=\frac{\tau}{\Delta t}$ 
Here, the residence time per unit length $\tau$ is the ratio between the average adatom concentration and the deposition rate $F$, and the time interval between two consecutive deposition events $\Delta t$ is $1 /[F(L-D)]$. The total nucleation rate is:

$w=(L-D) p F=\frac{F^{2}(L-D)^{4}}{6 v}$

The total nucleation probability $P$ is governed by $d P / d t=w(1-P)$. Under the condition of no extra energy barrier for the interlayer adatom transport, we have $D=F L t$. During the deposition of one ML, the space for nucleation goes from 0 to $L$, and the nucleation probability is:

$P=1-e^{-\int_{0}^{\frac{1}{F}} w d t}=1-e^{-\frac{F}{30 v} L^{4}}=1-e^{-\left(\frac{L}{L_{0}}\right)^{4}}$ with $\quad L_{0}=\left(\frac{30 v}{F}\right)^{\frac{1}{4}}$

The constant in the exponent, $L_{0}$, has been referred to as the critical length; for example, critical separation [3] and critical island size [9]. However, the measured critical separation may be slightly different from $L_{0}$. In the following, we will show that the difference is a factor of 0.91 . Based on the nucleation probability in equation (5), the nucleation probability density in $L$ space is:

$\frac{d P}{d L}=\frac{4 L^{3}}{L_{0}^{4}} e^{-\left(\frac{L}{L_{0}}\right)^{4}}$

Over a large ensemble, the measured value of the critical separation is:

$L_{S}=\int_{0}^{+\infty} L \frac{d P}{d L} d L=\Gamma\left(\frac{5}{4}\right) L_{0} \approx 0.91\left(\frac{30 v}{F}\right)^{\frac{1}{4}}$

where $\Gamma(x)=\int_{0}^{+\infty} e^{-t} t^{x-1} d t$ is the Gamma function. The critical separation so defined is the measured value and can be compared directly with computer simulation or experimental results. It differs from the constant $L_{0}$ by a factor of 0.91 ; in $3 \mathrm{D}$, this factor becomes 0.93 or $\Gamma\left(\frac{7}{6}\right)$. For consistency, we will from now on always refer the critical separation to mean the measured value as in equation (7), instead of the constant in the exponent as in equation (5). 
We note that the concept of nucleation probability as a function of length has been employed for the case of nucleation on an island. However, the concept had not been employed for the case of separation between nuclei, before our recent report [3]. In comparison with previous reports $[8,10,11]$, equation (7) gives the same scaling $\left(\frac{v}{F}\right)^{\frac{1}{4}}$. In contrast, equation (7) also gives the coefficient $0.91(30)^{\frac{1}{4}}$, which was previously unknown. The validity of equation (7), particularly the coefficient, will be verified using lattice KMC simulations.

\section{Lattice KMC simulations}

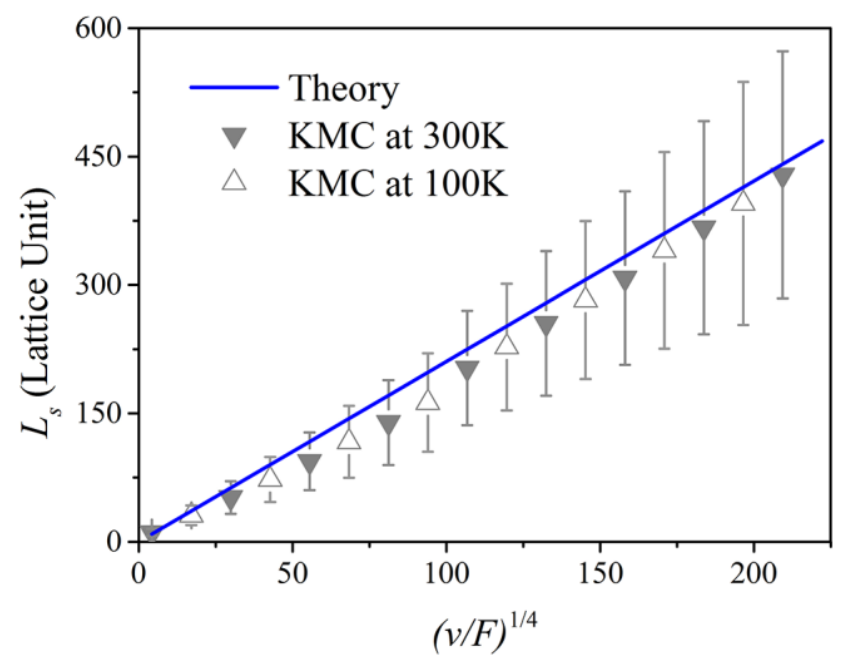

Fig. 3. Comparison of the closed-form theory and KMC simulation results, with the error bar representing the standard deviation.

We design the lattice KMC simulations to mimic the conditions of the analytical formulations for verification. Following similar KMC in our previous study [12], only atoms with one nearest neighbor diffuse and they represent the fastest diffusing adatoms. For self-consistent comparison and contrast between 2D and 3D results [3], we also use $\mathrm{Cu}\{111\}$ as the prototype surface in this study. For this surface, the diffusion jump rate of adatoms is $v=v_{0} e^{-E_{S} / k T}$, where $v_{0}$ is $5 \times 10^{11} / s$ and $E_{S}$ is $0.06 \mathrm{eV}$ according to ab initio calculations [13], and $k T$ is the Boltzmann factor in units of $\mathrm{eV}$. The substrate in the simulation has a length of 50000 
lattice units, corresponding to about $15000 \mathrm{~nm}$, the substrate temperature is $100 \mathrm{~K}$ or $300 \mathrm{~K}$, and the deposition rate $F$ is in the range of $10^{-2}-10^{5} \mathrm{ML} / \mathrm{s}$. For each set of deposition condition, we carry out at least 50 independent simulations, to have more than 2000 islands for convergence of results. As shown in Fig. 3, the simulation results agree with the closed-form theory, in terms of average. The huge standard deviations, particularly at large critical separations, might be perceived as lack of convergence of the simulation results. However, the large standard deviations are intrinsic to the distribution function in equation (6). According to this distribution function, the standard deviation is linearly proportional to the average value.

\section{Discussions and Conclusions}

Having derived and verified the closed-form theory, we now discuss the results to gain additional physical insights, and draw conclusions.
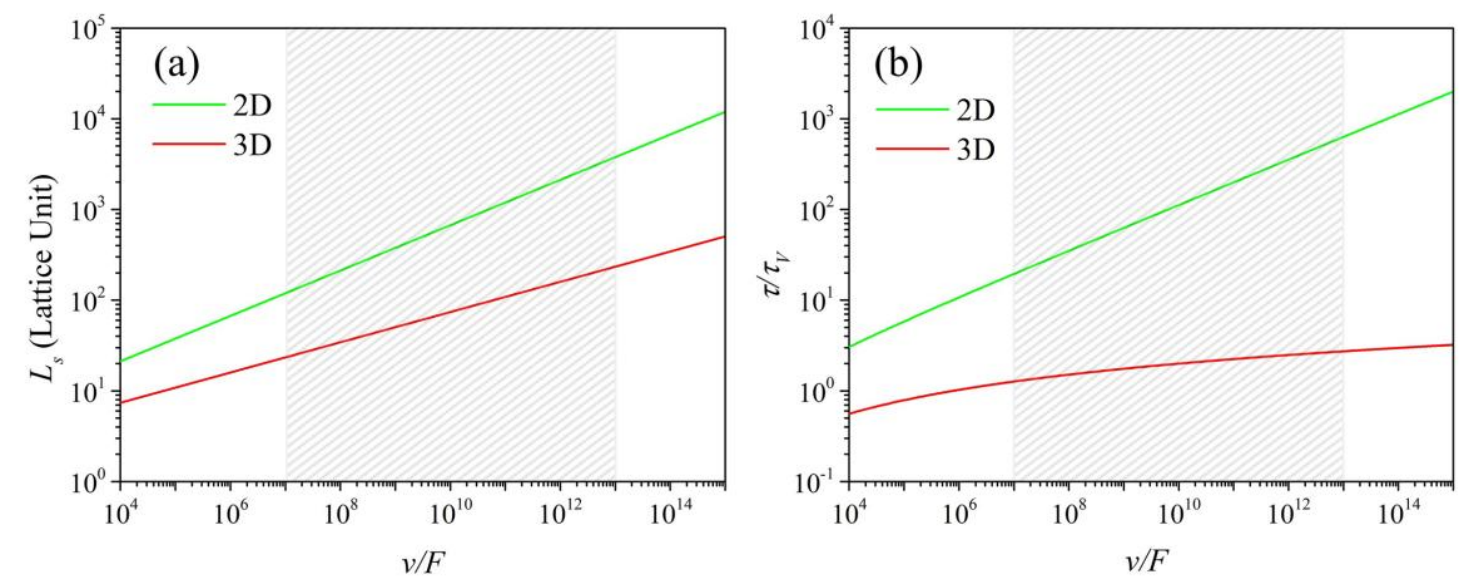

Fig. 4. Comparison of (a) the critical separation $L_{s}$ and (b) ratio $\tau / \tau_{V}$ as a function of $v / F$, in 2D and 3D for PVD on $\mathrm{Cu}\{111\}$.

As the first insight, we examine the order of magnitude of the critical separation $L_{S}$. For a range of typical copper PVD deposition conditions $-T \in$ $[200 K, 450 K], F \in\left[10^{-3} M L / s, 10^{5} M L / s\right]$, with $v / F$ being between $10^{7}$ and $10^{13}$ Fig. 4a shows that the separation of nuclei in $2 \mathrm{D}$ is about an order of magnitude larger than in $3 \mathrm{D}$. For the same diffusion jump rate, the larger separation in $2 \mathrm{D}$ will favor 
nanostructure formation or rougher surface; or the smaller separation in 3D will mean smaller surface roughness. This insight implies that reduction of surface diffusion anisotropy may enable the reduction of surface roughness during thin film deposition. In addition, this difference of $L_{s}$ in $2 \mathrm{D}$ and $3 \mathrm{D}$ also reveals that this length scale from 2D simulations is intrinsically off by an order of magnitude - this calls for caution in correlating 2D simulation results with 3D experimental counter parts.

As the second insight, we examine nucleation dynamics, which is dictated by two characteristic time scales. Qualitatively, it has been recognized that the nucleation dynamics in 2D and that in 3D differ [8]. However, with the closed-form theories, we now can appreciate this difference quantitatively, in terms of the range of effective diffusion $v / F$. The first time scale is the residence time of adatoms $\tau$, as defined early. The second time scale is the traversal time of adatoms $\tau_{V}=A / v$, where $\mathrm{A}$ is the area in $3 \mathrm{D}$ and length in $2 \mathrm{D}$ of the nucleation space.

When this ratio $\frac{\tau}{\tau_{V}} \gg 1$, majority of the time at most one adatom exists in the nucleation space. For example, if it takes one second for an adatom to traverse the entire nucleation space and the residence time is ten seconds, $\frac{\tau}{\tau_{V}}=10$. Within $10 \%$ of adatoms residence time, if there are two adatoms or more in the nucleation space, they will meet each other to form a nucleus or meet a nucleus - either way, at most only one adatom will remain during at least $90 \%$ of the adatoms residence atom. This is the criterion for LAM to be valid [9]. On the other hand, when $\frac{\tau}{\tau_{V}} \sim 1$, multiple adatoms can co-exist in the nucleation space and the conventional nucleation theory applies. As shown in Fig. 4b, under typical PVD conditions LAM applies in 2D since $\frac{\tau}{\tau_{V}}>$ 10 , but the conventional nucleation theory applies in $3 \mathrm{D}$ since $\frac{\tau}{\tau_{V}} \sim 1$. It should be noted that LAM applies even in 3D when the area of nucleation is very small, as is the case on top of a small nanorods [4].

In conclusion, we report a closed-form theory for the critical separation of 
nuclei in $2 \mathrm{D}$. Based on this new theory and the previously reported theory in $3 \mathrm{D}$, the critical separation of nuclei on a diffusion anisotropic surface is about an order of magnitude larger than on a diffusion isotropic surface. Further, this theory allows one to quantitatively compare and contrast the nucleation behavior on surfaces of various diffusion anisotropy; with isotropic surfaces governed by the 3D theory, and extremely anisotropic surfaces governed by the 2D theory.

Acknowledgement: The authors acknowledge financial support from the Department of Energy Office of Basic Energy Sciences (DE-SC0014035).

\section{References:}

1 L.H.J. Tang, Island formation in submonolayer epitaxy. Phys. I France 3, 935 (1993).

2 M.F. Gyure, C. Ratsch, B. Merriman, R.E. Caflisch, S. Osher, J.J. Zinck, D.D. Vvedensky, Level-set methods for the simulation of epitaxial phenomena. Phys. Rev. E 58, R6927 (1998).

3 L.G. Zhou, H.C. Huang, Smallest separation of nanorods from physical vapor deposition. Appl. Phys. Lett. 100, 141605 (2012).

4 X. Niu, S.P. Stagon, H.C. Huang, J.K. Baldwin, A. Misra, Smallest metallic nanorods using physical vapor deposition. Phys. Rev. Lett. 110, 136102 (2013).

5 Y.W. Mo, J. Kleiner, M.B. Webb, M.G. Lagally, Activation energy for surface diffusion of $\mathrm{Si}$ on $\mathrm{Si}(001)$ : A scanning-tunneling-microscopy study. Phys. Rev. Lett. 66, 1998 (1991).

6 P. Politi, Different regimes in the Ehrlich-Schwoebel instability. J. Phys. I France 7, 797 (1997).

7 J. Tersoff, A.W. Denier van der Gon, R. M. Tromp, Critical island size for layer-by-layer growth. Phys. Rev. Lett. 72, 266 (1994).

8 A. Pimpinelli, J. Villain, D.E. Wolf, Surface diffusion and island density. Phys. Rev. Lett. 69, 6 (1992).

9 J. Krug, P. Politi, T. Michely, Island nucleation in the presence of step-edge barriers: Theory and applications. Phys. Rev. B 61, 14037 (2000).

10 H. Kallabis,, P.L. Krapivsky, D.E. Wolf, Island distance in one-dimensional epitaxial growth. Eur. Phys. J. B, 5, 3 (1998).

11 J. Villain, A. Pimpinelli, L. Tang, D. Wolf, Terrace sizes in molecular-beam epitaxy. J. Phys. I France 2, 2107 (1992).

12 L.G. Zhou, H.C. Huang, Characteristic length scale of nanorod diameter during growth. Phys. Rev. Lett. 101, 266102 (2008).

13 S.K. Xiang, H.C. Huang, Ab initio determination of Ehrlich-Schwoebel barriers on $\mathrm{Cu}\{111\}$. Appl. Phys. Lett. 92, 141605 (2008). 